\title{
Colonialismo: un episodio en la Historia Africana
}

\author{
JAKOB F. AdE AJAYI ${ }^{*}$
}

* TITLE AND AUTHOR OF THE LICENSED MATERIAL: PUBLICADO COMO AIAYI, J.F.ADE, "COLONIALISM: AN EPISODE IN AFRICAN HISTORY”, EN GANN, LEWIS.H.Y DUIGNAM, PETER. (EDS.). COLONIALISM IN AFRICA 1870-1960. VOL. I. THE HISTORY OF POLITICS OF COLONIALISM I870-19I4. CAMBRIDGE, CAMBRIDGE UNIVERSITY PRESS, 1969.

THIS MATERIAL HAS BEEN PUBLISHED IN JOURNAL RELACIONES INTERNATCIONALES TRANSLATED BY ALICIA CAMPOS SERRANO.THISVERSION IS FREETOVIEW AND DOWNLOAD FOR PRIVATE RESEARCHAND STUDY ONLY. NOT FOR RE-DISTRIBUTION OR RE-USE. C

REPRODUCED WITH PERMISSION OFTHE LICENSOR THROUGH PLSCLEAR.

Traducción:

Alicia Campos Serrano, Universidad Autónoma de Madrid, Departamento de Antropología Social

\section{Presentación del texto, por Alicia Campos Serrano UNIVERSIDAD AUTÓNOMA DE MADRID \\ Departamento de Antropología Social \\ Contacto: alicia.campos@uam.es}

La publicación en español de un texto de 1969 sobre el colonialismo en África requiere de una justificación, aunque sea pequeña. En aquellos años, la descolonización era un proceso en curso que ya había dado lugar a numerosos estados independientes. Y jóvenes historiadores, fuera y dentro del continente, trabajaban con entusiasmo por ofrecer una "historia africana", alejada de los presupuestos hegelianos y eurocéntricos que habían negado, en las décadas anteriores, la historicidad de las poblaciones al sur del Sáhara. Eran tiempos de "construcción de naciones", y había que proporcionar historias donde los africanos aparecieran como protagonistas, hacedores de estados y sistemas comerciales, y no como meras víctimas o elementos del paisaje.

Medio siglo más tarde, la Historia de África es ya una disciplina consolidada que, con sus dificultades y debates, se sitúa al mismo nivel de la que se hace sobre otros lugares del mundo (Coquery-Vidrovitch, 20I3-20I4). La producción, los temas y los ámbitos de trabajo de los historiadores africanistas se han multiplicado desde la era de las independencias, y los ánimos nacionalistas que inspiraron a los pioneros también se han calmado. Pero la recuperación en castellano de un texto seminal como este tiene sentido en nuestro contexto por el doble objetivo que busca.

\section{DOI:}

http://doi.org// 0.15366/relacionesinternacionales2020.44.009

Formato de citación recomendado:

AJAYI, Jakob F.Ade (2020), “Colonialismo: un episodio en la historia de África”, Relaciones Internacionales, $\mathrm{n}^{\circ}$ 44, pp. 165-176. 
En primer lugar, queremos dar a conocer, entre estudiosos habitualmente más centrados en el mundo latinoamericano, a los pioneros de una historiografía a menudo ignorada por estos lares, pero que habla de temas afines y sobre una región con intensos vínculos atlánticos. Por otra parte, pretende contribuir al renovado debate propuesto por las corrientes de los estudios subalternos, poscoloniales o decoloniales, recordando que el esfuerzo por "deseurocentrar" la historia y dar voz a los "grupos subalternos" (véase por ejemplo Chakrabarty, 2008), no ha dejado de ser una constante entre los historiadores africanistas desde los años 1950. El énfasis en la "iniciativa africana", la insistencia en que los africanos eran autores de su propia historia, las ideas de "resistencia" y de "reacciones" ante la invasión colonial europea se convirtieron en leitmotivs de las primeras escuelas, que tuvieron en lbadán, Dar-Es-Salám y Dakar, así como en algunas universidades europeas, centros fundamentales de desarrollo.

No obstante, autores como Ade Ajayi (1929-2014) fueron más allá de lo que hoy ya resulta obvio: que la historia de África es mucho más que la historia europea a la que se suma la reacción de los africanos. En textos como el que aquí se presenta, Ajayi propuso una mirada sobre la propia dominación colonial desde finales del siglo $X X$, que constituye un verdadero programa de investigación todavía con vigencia. Desde esta mirada, los europeos no aparecen como gobernantes todopoderosos, sino como actores enfrentados a limitaciones similares a que las que encontraron autoridades africanas antes que ellos para construir órdenes políticos centralizados.

El impacto del reparto y dominio europeos sobre los órdenes sociales y políticos previos resulta incuestionable. Pero en este proceso, los africanos no aparecen como poblaciones homogéneas e inertes ante la acción de civilizadores y explotadores: lo que Ajayi nos presenta son situaciones sociales complejas donde individuos y grupos en posiciones sociales distintas adoptaron una diversidad de actitudes ante los nuevos contextos. No caben para nuestro autor dicotomías simplificadoras como resistencia y colaboración, en la medida en que ambas pueden formar parte de estrategias múltiples y cambiantes de unos mismos actores a lo largo del tiempo. Tampoco la historia previa queda interrumpida: más bien condiciona las formas concretas que fue adquiriendo aquel dominio. Dinámicas sociales, relaciones y conflictos venidos del periodo anterior continuaron bajo los regímenes coloniales, que no pueden dar cuenta por sí solos de todos los procesos sociales y políticos que siguieron desarrollándose en el continente mientras duraron.

Con esta sensibilidad hacia las tensiones y la complejidad histórica, la obra de Ajayi se extiendió a otros ámbitos: sus trabajos sobre las guerras yoruba o las misiones cristianas en Nigeria, en las décadas previas al reparto colonial, siguen siendo hoy referentes entre los especialistas. Su rigor en la exploración y uso de nuevas fuentes y métodos historiográficos, que cubriesen las limitaciones de las fuentes archivísticas para la historia de África, le valió un amplio reconocimiento internacional.Tuvo además un papel fundamental en la consolidación de la academia nigeriana, y en especial de la Universidad de lbadán, desde donde desarrolló una intensa labor como docente y promotor de jóvenes académicos. Su actividad como editor de la revista Journal of the Historical Society, la colección de trabajos Ibadan History Series o el volumen VI de la Historia General de África de la UNESCO, sobre el siglo XIX hasta 1880, dan cuenta de su compromiso por la promoción y la divulgación de la Historia de África, así como de los historiadores africanos. 


\title{
Lecturas de apoyo recomendadas por la traductora
}

Falola, T. (1993). African Historiography. Essays in honour of Jacob Ade Ajayi. Ikeja: Longman Nigeria.

Chakrabarty, D. (2008). Más allá de Europa. Pensamiento poscolonial y diferencia histórica. Barcelona:Tusquets.

Coquery-Vidrovitch, C. (20I3-20 I4). L’historiography africaine en Afrique. Revue Tier Monde, 216.

Bown, L. ( 10.09 .20 I4).JFAde Ajayi obituary. Pioneer in the study of African history who highlighted native perspectives and the complexities of colonial-era change. The Guardian. Recuperado de: https://www.theguardian.com/ books/20I4/sep/I0/jf-ade-ajayi (30.05.2020)

Ade Ajayi, J.F. (Ed.). ( 1989 ).Africa in the nineteenth century until the I880s. UNESCO General History of Africa,VolVI. California:James Currey. Recuperado de: https://unesdoc.unesco.org/ark:/48223/pf0000 I 34378 (30.05.2020)

\section{Colonialismo: un episodio en la historia africana Por J. F.A.AJAYI}

\begin{abstract}
ir Harry $\mathrm{H}$. Johnston fue uno de los constructores de imperios europeos más sofisticados. Veía la invasión y conquista europea de África a finales del siglo XIX, no como un acontecimiento aislado, sino como la última de toda una serie de invasiones desde los fenicios, los griegos y los romanos, hasta los árabes, los turcos y otras de medio y lejano Oriente. La concebía, además, como la culminación de las actividades de los europeos en África iniciadas por las exploraciones portuguesas en el siglo XV. Su History of the colonization of Africa by alien races fue un esfuerzo pionero de ver el reparto de África en una perspectiva temporal, y es por tanto un punto de partida útil para este debate sobre el significado del colonialismo europeo en la historia africana (Johnston, I9I3).
\end{abstract}

Johnston no compartía la ilusión romántica de muchos de sus contemporáneos, particularmente aquellos responsables militares de las "expediciones de castigo", que se veían a sí mismos como nuevos conquistadores en posición de dioses destinados a exterminar a las gentes atrasadas o decadentes y desbrozar el terreno para una civilización totalmente nueva. Sin embargo, compartía la creencia victoriana de que los europeos no eran como otros hombres, y que entre toda la humanidad, eran "las subespecies de piel blanca las únicas que habían desarrollado belleza en los rasgos faciales y originalidad en la invención de ideas y de hechos" (Johnston, 1913, p. 450).

Veía, por tanto, la intervención de los europeos en los asuntos africanos con una finalidad histórica de la que habían carecido las invasiones previas, una finalidad histórica como la que la historiografía cristiana sólo reserva a la intervención divina. Creía que los europeos eran portadores de una nueva civilización, y que estaban destinados a dejar su marca en la naturaleza física y mental del hombre en África. 
No necesitamos tener ninguna consideración con estas pintorescas ideas, excepto como representación de un aspecto de la colonización en el periodo entre el reparto y la Primera Guerra Mundial que tiende a ser ignorado. Si antes y después del Congreso de Berlín existió continuidad en la implicación europea, especialmente en África Occidental, la transformación de un imperio informal en uno formal necesitó de cambios de actitud significativos por parte de los europeos. No hubo sólo un aumento del sentimiento racial (Ajayi, 1965), sino también de la creencia de que la colonización debía ser idealmente como la practicada en las Américas y Australasia, que implicó la inmigración, el asentamiento y el mestizaje europeos en alto grado. Johnston se dio cuenta de que esto sólo era factible en zonas templadas y en las tierras altas. Mantuvo que las áreas tropicales, donde el clima y las enfermedades dificultaban el asentamiento europeo a gran escala, la presencia de africanos no podía ser ignorada. Pero esto no destruyó su convicción de que el gobierno europeo en África tendría efectos raciales y demográficos permanentes. Era posible, decía, que la ciencia pudiera "anular los efectos insanos de un clima tropical" o que "pudiera irrumpir una nueva enfermedad que destruyera al negro y dejara sólo al hombre blanco" (Johnston, 1913, pp. 445 y 450). Pero incluso sin tales posibilidades extremas, creía que el colonialismo europeo afectaría de algún modo a los rasgos físicos de los africanos. "Sin duda", decía, "como en Asia y Suramérica, el eventual resultado de la colonización de África por pueblos extranjeros será un compromiso entre una raza oscura con los rasgos y el cerebro del hombre blanco" (Johnston, 1913, p. 45I)².

Más allá de esta obsesión racista por los rasgos físicos, Johnston veía el establecimiento del gobierno colonial como el comienzo de un largo proceso de educación de los africanos en las tecnologías de una nueva civilización.Ya que a corto plazo no era posible el asentamiento europeo a gran escala, y que la presencia africana no podía ignorarse, los africanos deberían ser instruidos, por los asiáticos si fuera necesario, para tomar la iniciativa de su propio desarrollo. Preveía un prolongado proceso de tutela que se mediría en siglos, pero no descartaba una final iniciativa africana. En la edición del libro de 1899 , decía:

Así como sería necesario algún acontecimiento asombroso y extraordinario para que toda Asia se levantara como un solo hombre contra la invasión de Europa, no es difícil imaginar que el hombre negro formará con el tiempo un pueblo negro unido que exija autonomía, poniendo fin al control del hombre blanco y a la inmigración, asentamiento y mezcla de las razas superiores de Europa y Asia.

"En la edición de 1913, añadió con respecto a la de 1899 (Johnston, 1899, p. 284) que esta concepción era "Difícil... pero no imposible... Cualquier predicción sobre el futuro de Continente Negro parece inútil a la luz de lo inesperado, lo extraño, lo imprevisto de lo que surge la misma África” (Johnston, I913, p. 450).

Hemos llegado mucho más lejos que Johnson en nuestro acercamiento al estudio de la historia africana; ahora sabemos que su análisis era incorrecto porque la intervención europea se ha demostrado mucho menos permanente de lo que él pensaba que sería. Johnson no creía que los africanos tuvieran una historia más allá de las actividades de los invasores europeos y asiáticos. Aunque nuestro conocimiento de la historia interna de África es todavía muy fragmentario, pocas

2 Compárese la mirada misionera de que “los africanos deben convertirse en un nuevo hombre” (Groves, 1969, p. 455). 
personas responsables negarán hoy que el "Continente Oscuro" tiene, de hecho, una historia que necesita ser estudiada y comprendida. En nuestro estudio de las iniciativas europeas en África somos ahora más conscientes de la presencia de los africanos, y prestamos atención a sus reacciones a las actividades europeas. Pero todavía tendemos a pensar que podemos comprender el colonialismo y sus consecuencias para los pueblos africanos básicamente estudiando el impacto europeo y las reacciones de los africanos a las mismas, sin relacionarlas con la historia interna de África y de los africanos.

Una evaluación completa de los efectos del colonialismo sobre África y sobre los pueblos africanos debe, sin embargo, hacerse en un contexto histórico. Esto ya lo decía Johnson. Pero este contexto histórico no es la historia de la colonización de África o la historia de las reacciones africanas a la colonización europea, sino la historia africana misma. Como el mismo Johnson dijo en un momento de sensibilidad histórica, los factores que probablemente afectaron al devenir del colonialismo no fueron sólo externos como solemos enfatizar — por ejemplo, las guerras mundiales, la revolución rusa y el nacionalismo en el sureste asiático- sino "lo inesperado, lo extraño, lo imprevisto de lo que surge la misma África". Sólo necesitamos añadir que las llamadas reacciones africanas a las actividades europeas aparecen inesperadas y extrañas sólo cuando no se examinan sus raíces en la historia africana.

\section{La continuidad de la historia africana}

El reparto colonial debe ser comprendido, por tanto, en el contexto del conjunto de la historia de África. El siglo XIX es probablemente el periodo mejor conocido, y la conquista europea está ahora bien situada en el escenario general de la historia africana del siglo XIX.Visto en el contexto de las revoluciones islámicas en el Sudán, las migraciones bantúes y los intentos de formación estatal de Shaka, Moshes y otros, o las diferentes guerras y acomodaciones que ocurrieron en las áreas costeras de África Occidental a lo largo del siglo, el reparto ya adopta una forma más inteligible. Ahora es posible escribir la historia de la conquista y el establecimiento del gobierno europeo en África en términos de la interacción de dos conjuntos de seres humanos, más que en términos de la visión de la época de los europeos como dioses que trataban con nativos subhumanos.

Sin embargo, como conocemos menos sobre los siglos anteriores, existe una tendencia a asumir que el siglo XIX fue inusualmente activo en la historia africana, como si hubiera sido configurado especialmente de esa manera para preparar el camino al reparto europeo. De hecho, el profesor Hargreaves habla de "una especie de reparto africano de África, una reordenación radical de estructuras y fronteras políticas que tuvo lugar a lo largo del siglo" (Hargreaves, 1969, p. 199). Separada del resto de la historia africana, el siglo XIX comienza a parecer un periodo en el que los africanos mismos salieron de su camino habitual para anticipar la gran invasión europea. Pero ¿podemos asumir esto?

De manera alternativa, a veces se sugiere que el incremento del ritmo de las actividades africanas en el siglo XIX fue una reacción directa al aumento del ritmo de las actividades europeas anteriores al reparto. Colson mantiene esta visión y se inclina a observar los principales desarrollos históricos en África desde la era de la trata como una reacción directa de las maniobras europeas, siendo estos desarrollos de mayor alcance y significación en África Occidental que en África 
Oriental en proporción a la intensidad de la implicación europea (Colson, 1969, p. 27). Este argumento exagera la medida en que las actividades de los europeos pueden ser vistas como los acontecimientos centrales de la historia africana, de las que todas las demás derivan. Equipara las reacciones de los africanos a la iniciativa europea con la totalidad de la historia africana o, más bien, niega la iniciativa africana a lo largo de la historia africana. El hecho de que tengamos conocimiento, y con frecuencia datos concretos, sobre el comercio europeo, y a menudo información menos específica acerca de la historia de las comunidades de África Occidental, no justifica la idea de que el surgimiento y caída de los estados en esta región deba ser explicado fundamentalmente en términos de la trata de esclavos (véase por ejemplo Ade Ajayi y Smith, 1964, pp. 123- 127). Esto supone desplazar la frontera de las actividades europeas en el siglo XIX hacia áreas del interior demasiado amplias, y enfatizan excesivamente el avance europeo, antes de que los europeos comenzaran realmente a tomar la iniciativa en la conformación de la historia africana. Incluso así, la pregunta continúa siendo cuánta iniciativa conservaron los africanos durante el reparto cuando la explotación europea se volvió más intensa.

Para observar el reparto dentro de la perspectiva adecuada de la historia africana, tenemos por tanto que mirar más allá del siglo XIX y más allá de los principales centros de actividad europea. Igual que ahora enfatizamos que las sociedades africanas precoloniales no permanecieron estáticas hasta que los europeos comenzaron a alterarlas en el periodo colonial, deberíamos enfatizar también que no eran estáticas antes de la llegada de las revoluciones islámicas o los abolicionistas del siglo XIX. Tampoco habían sido estáticas antes de la llegada de los holandeses en el siglo XVII o de los portugueses en el XV. Existieron pueblos africanos como los Yoruba y los Edo de Nigeria austral que habían organizado estados en respuesta a su entorno, sus necesidades económicas y la calidad de su liderazgo antes de la llegada de los europeos. En la historia de dichos estados, la entrada de los europeos en escena, y el creciente ritmo de sus actividades, fueron sólo incidentales. No deberíamos dar por hecho que el factor colonial asumió un papel dominante sobre todos los demás de los que habían afectado previamente a su historia, como la ecología, los factores económicos no relacionados con el comercio europeo, la calidad del liderazgo, o los problemas u oportunidades políticas en diferentes periodos. Tampoco hay necesidad de asumir que todos esos elementos no estaban presentes o eran siempre subsidiarios del factor europeo en el surgimiento, la naturaleza o la caída de los estados que se han desarrollado en África desde la llegada de los europeos. Incluso en el caso de estados tan dependientes del comercio europeo como las ciudades estado del Delta (del Níger) a inicios del siglo XIX, la evidencia parece indicar que el impacto del comercio europeo en su desarrollo estructural se ha exagerado enormemente (Jones, 1963).

\section{El impacto colonial}

Sostener que los principales movimientos políticos en la historia africana no estuvieron necesariamente inspirados por las iniciativas europeas no supone negar que los factores externos han tenido un impacto importante. Las interrelaciones entre los factores internos y externos generarán debate durante mucho tiempo. La historiografía reciente, reflejada tan ampliamente a lo largo de este libro, se ha esforzado en enfatizar que, particularmente en el siglo XIX, los líderes políticos y religiosos africanos retuvieron en gran medida la iniciativa en la dirección de los asuntos africanos a pesar de la creciente intensidad del papel de los europeos. Existe, sin embargo, el riesgo de inclinar en exceso el equilibrio cuando se sugiere que el reparto fue provocado en gran medida 
por un creciente nacionalismo en África, que amenazó con limitar las empresas europeas en los imperios informales, forzando a los europeos a establecer su dominio formal. Igualmente, aunque es cierto que las revueltas africanas contra los gobiernos europeos mostraron cuán vulnerables eran, y qué mal provistos estaban de personal, es probablemente una exageración sugerir que los europeos no fueron en general dueños de la situación colonial. La definitiva superioridad de las armas europeas fue una realidad insoslayable, y la división entre los africanos, o el poder del mito del “invencible hombre blanco”, no pueden ser ignorados por completo.

El aspecto fundamental del impacto europeo fue la pérdida de soberanía que conllevó para prácticamente todos los pueblos africanos. Los europeos explotaron su superioridad tecnológica para establecer su dominio político a lo largo del continente.A menudo reprimieron, suprimieron o amalgamaron estados a voluntad. En algunos casos establecieron un gobierno directo con el objetivo consciente de organizar la vida cotidiana de los africanos. En otras áreas, se permitieron diversos grados de autonomía local. Los regímenes coloniales sufrieron de la debilidad de todas las administraciones que carecen de apoyo popular. Pero su habilidad final de suprimir la rebelión era indudable. Fueron capaces de ejercer ampliamente un completo aunque desigual control, arbitrario e irresponsable.

Tal vez la dimensión más significativa fue el alcance que tuvo el mismo reparto del continente. Los territorios en los que África fue dividida marcaron dinámicas totalmente nuevas en la historia de África. En cierto sentido, los nuevos territorios eran sucesores de imperios, estados y reinos africanos anteriores, pero con una importante diferencia. Las fronteras de los antiguos imperios se expandían o retrocedían a voluntad. Nada era permanente en el flujo y reflujo de la historia. Pero el acto europeo del reparto intentó detener estas fluctuaciones mientras canalizaba el desarrollo en direcciones enteramente inusitadas. Las nuevas fronteras, una vez que los mismos europeos se habían puesto de acuerdo sobre ellas, se pretendían permanentes y ya no se expandieron o retrocedieron a voluntad de los africanos. Se establecieron además líneas de separación entre grupos humanos, con un desprecio total por los destinos históricos de comunidades hasta entonces contiguas y algunas veces muy relacionadas. Durante mucho tiempo, e incluso ahora con la excepción de unas pocas, especialmente en los territorios bajo mandato (de la Sociedad de Naciones) ${ }^{3 *}$, estas fronteras han sido vistas como no negociables. En ese sentido al menos, la intervención europea ha tenido, por el momento, algo de la finalidad que le atribuía Johnston.

Esta soberanía política fue la base para la dominación cultural europea. A través de la misma, el cristianismo, la educación occidental, las ideas sociales y políticas occidentales, tuvieron una profunda influencia sobre las instituciones africanas. El impacto del cristianismo no se limitó a aquellos que se convirtieron o cayeron bajo la influencia directa de la Iglesia. Los regímenes coloniales utilizaron su soberanía para suprimir sin piedad prácticas que eran incompatibles con las tradiciones cristianas de la sociedad occidental. Con ello, los nuevos gobernantes debilitaron la confianza de la gente en los antiguos dioses y en el antiguo orden social. Promovieron el escepticismo científico sobre la intervención directa de las fuerzas supranaturales en la vida social, y de esta forma tendieron a debilitar la fe en las sanciones tradicionales que mantenían a la sociedad unida.

\footnotetext{
*Aclaración de la traductora.
} 
El dominio colonial fomentó una campaña contra las costumbres del matrimonio polígamo y la familia extensa; y trabajó por el acercamiento individualista y exclusivamente patrilineal del mundo occidental. De igual manera, la esclavitud y el comercio de esclavos fue suprimido, con consecuencias sociales, económicas y políticas de largo alcance. Nuevas orientaciones económicas, la minería y la industrias, las carreteras y vías de tren y la educación occidental promovieron el crecimiento de las ciudades. Instituciones africanas tradicionales que tenían sus raíces en los pueblos y la vida rural tuvieron que transformarse y adaptarse a las pautas de la vida urbana.

Por muy amplios que estos cambios puedan haber sido, su impacto sobre África fue muy desigual. Mientras que las vidas de algunas comunidades se vieron profundamente afectadas, otras apenas fueron conscientes de la presencia de los europeos antes de que comenzaran a marcharse. Más aún, los regímenes coloniales estuvieron lejos de ser radicales. Así como las fronteras de los territorios coloniales tendieron a poner un freno en el cambio histórico, los gobiernos coloniales tendieron a aliarse con los elementos más conservadores de la sociedad y a detener el proceso normal de cambio social y político. Una vez que la conquista se había conseguido, fueron los jefes sometidos, los guardianes de la ley, el orden y las costumbres sagradas, más que la nueva élite educada, quienes se convirtieron en los agentes favorecidos por la administración europea. Ningún régimen colonial habría dudado en aliarse con las fuerzas más conservadores para derrocar a un líder hostil aunque fuera progresista y modernizador. Después de todo, la principal preocupación de aquellos regímenes no era llevar a cabo reformas sociales sino sobre todo controlar y mantener la ley y el orden para facilitar la explotación económica. Es verdad que para controlar tuvieron que hacer uso de fuerzas aparentemente revolucionarias en la sociedad, así como deponer gobernantes conservadores hostiles. Pero su sucesor, una vez llegado al poder, tendría que abstenerse de llevar a cabo demasiadas reformas sociales. De otro modo, correría el riesgo de perder el apoyo del gobierno colonial cuyo interés principal, repetimos, era el mantenimiento de la ley y el orden, no la reforma. El resultado ha sido, por tanto, que muchos de los gobiernos nacionalistas se encuentran hoy con que en muchos asuntos tienen que tomar los hilos de la reforma social y política en el punto en el que los reformistas musulmanes y cristianos del siglo XIX los dejaron a la llegada del gobierno colonial.

Por tanto, aunque los europeos fueron generalmente señores de la situación colonial y disfrutaron de la soberanía política y el dominio cultural y económico, no poseyeron el monopolio de la iniciativa durante el periodo colonial. En la medida en que los africanos retuvieron la iniciativa, la habilidad de los europeos para hacer grandes transformaciones en la historia africana fue limitada.

\section{Iniciativa africana}

A este respecto, ha aumentado recientemente el interés sobre los movimientos de resistencia. La historiografía de los movimientos de resistencia ha prestado recientemente mucho interés a esta conexión. Estos movimientos son interesantes no sólo en la medida en que muestran la valentía africana, un fuerte nacionalismo o un especial talento para la organización militar, comparable con la de los europeos. Más destacable incluso es la amplia variedad de reacciones por parte de diferentes comunidades africanas, cada una enfrentada con el reto de la conquista y la supremacía europea. 
Un hecho que merece subrayarse es que los movimientos de resistencia no pueden ser considerados sólo para distinguir a los pueblos patrióticos y valientes deseosos de oponerse a la agresión, de los tibios y oportunistas deseosos de colaborar con los invasores. La cuestión de la paz o la guerra debe observarse más bien como un aspecto de la política externa de cada comunidad africana. Las decisiones africanas dependían no sólo de los intereses similares o en conflicto en un determinado momento de un estado africano particular con el poder invasor, sino también de la relación con los estados africanos vecinos, cuyas actitudes hacia dicho invasor era a menudo un factor crucial. Esto de nuevo enfatiza que cada conquista europea, incluyendo las diversas reacciones de los africanos a los poderes colonizadores, no pueden ser totalmente comprendidos excepto en el contexto de una historia africana anterior.

Cada organización africana — pueblo, ciudad o reino — veía el reto de la conquista europea como un factor histórico nuevo. Los africanos podían resistir al hombre blanco, formar alianzas con los recién llegados, o explotarlos en la medida de lo posible en una lucha continua por la supervivencia, la riqueza o el poder. Como guardianes de la soberanía del pueblo, los gobernantes de estos estados fueron normalmente hostiles a cualquier poder que pusiera en cuestión esta soberanía. Alguno de ellos creyó lo que contaban al inicio, entre otros, los misioneros: que la presencia europea no amenazaba, en realidad, su soberanía. Algunos de los estados más débiles, hostigados por sus vecinos, dieron la bienvenida al dominio europeo como una forma de liberación. Varias comunidades aceptaron cooperar o aliarse con los invasores, y después se volvieron belicosos cuando descubrieron la extensión de las ambiciones europeas. Otros cambiaron de la hostilidad a la cooperación tras aceptar la derrota o reconocer una fuerza superior. Estos aspectos son debatidos en profundidad por los profesores Ranger y Hargreaves en sus respectivos ensayos sobre los movimientos de resistencia en África Oriental y África Occidental. Estos cambios de actitud eran normalmente resultado de los cambios de intereses de la comunidad en su conjunto; pero a veces también reflejaban actitudes divergentes entre diferentes clases o líderes dentro de la misma comunidad. Así, la historia de las reacciones al dominio extranjero reflejó a veces cambios internos en la estructura de poder de la comunidad.

De especial importancia para determinar la habilidad de las comunidades para explotar las oportunidades del periodo colonial fue la existencia de una élite con una educación occidental, que era a su vez dependiente de la educación misionera, así como de la receptividad de la comunidad al empeño de las órdenes religiosas. La élite educada estaba motivada por consideraciones diferentes a las de los gobernantes tradicionales. Aquellos expresaron una hostilidad general a la imposición del gobierno europeo; y algunos de sus escritos, que mostraron temores exagerados a que el gobierno europeo significara el fin de los africanos como pueblo diferenciado, reprodujeron de hecho las actitudes racistas de los invasores. ${ }^{4}$ Sin embargo como grupo fueron los más dispuestos a ver en el aumento de las actividades europeas una gran oportunidad que aprovechar. Como ha dicho el Profesor Flint, tal vez con alguna exageración, "es una ironía histórica que las únicas personas que en la víspera del reparto de África poseían algún sentido de "misión imperial" británica fueron africanos educados o semi-educados" (Flint, 1969, p. 222).

\footnotetext{
4 Por ejemplo: "Tal vez el mundo nunca ha sido testigo hasta ahora de una rapiña a tan gran escala. África está indefensa para evitarlo... Está sobre la mesa que este negocio "cristiano" sólo puede llevar, en un futuro no lejano, a la aniquilación de los nativos” (Lagos Observer, I 885 sobre la conclusión de la Conferencia de Berlín sobre África Occidental).
} 
Como comerciantes, veían en la extensión del dominio europeo un aumento inmenso de oportunidades comerciales. Como profesores y oficinistas, esperaban oportunidades de trabajos. Como visionarios de nuevas naciones africanas, miraban la construcción de carreteras, vías férreas, puertos, hospitales, escuelas y otras características de la civilización tecnológica europea como la infraestructura necesaria de las naciones de sus sueños. Estaban de acuerdo con los invasores en que la tutela europea permitiría a los africanos tomar la iniciativa en su propio desarrollo. Pero como nunca habían dudado de la capacidad africana para autogobernarse, no esperaban que el periodo de tutela fuera largo. En la medida en que eran conscientes de la continuidad de la historia africana, no percibieron en un inicio la finalidad que Johnston y otros veían en la intervención europea, o los temores que la mayoría de la élite tradicional tenía respecto a una pérdida de soberanía africana, aunque fuera temporal. Pronto tendrían que cambiar sus opiniones; y mientras la élite tradicional lideraba los movimientos de resistencia inicial contra el gobierno colonial, los "hombres nuevos" liderarían las protestas nacionalistas que les siguieron.

La iniciativa africana, sin embargo, no se limitaba a estas resistencias y movimientos nacionalistas. Se desplegó también en innumerables presiones y maniobras locales, cada una pequeña en sí misma, pero que tuvieron el efecto total de limitar considerablemente el alcance del control de los regímenes europeos a lo largo de todo el periodo colonial. Un ejemplo significativo de esto es que las fronteras de distrito dentro de los territorios coloniales nunca consiguieron la permanencia de las fronteras nacionales.

Mientras los administradores coloniales buscaron las unidades más racionales de gobierno, se veían sometidos a las presiones de las comunidades locales para que las nuevas fronteras se adaptaran a intereses políticos de diferentes comunidades, o a factores históricos previos, ignorados hasta el momento por los gobernantes extranjeros. Los británicos, por ejemplo, cedieron a esas reclamaciones tanto en Uganda como en el norte de Rodesia. Estos intereses políticos y factores históricos fueron un aspecto de la historia de las relaciones intergrupales, y su importancia en el periodo colonial es otra muestra de la continuidad no interrumpida de la historia africana desde el periodo precolonial (véase por ejemplo Stahl, 1964). Porque dentro de las fronteras internacionales que crearon los europeos, y ocasionalmente a través de ellas, diferentes comunidades continuaron sus pautas históricas en la búsqueda de la supervivencia y el desarrollo.

Decisiones europeas arbitrarias sobre la localización de las vías férreas, los puertos, las escuelas, los hospitales y los centros administrativos afectaron la fortuna de estas comunidades; hasta este punto la iniciativa europea fue substancial. Pero hasta donde estas decisiones podían verse afectadas por los africanos mismos a través de la guerra, las revueltas, las protestas o las empresas e iniciativas locales para producir un personal cualificado, los africanos también retuvieron cierto control sobre sus propios destinos.

Estos esfuerzos e iniciativas representan sólo en parte una reacción a las actividades europeas.También son parte de la historia de desarrollo interno de las comunidades y las relaciones intergrupales africanas en la historia africana. Esta es la razón de que el impacto colonial no pueda ser completamente comprendido o analizado excepto en el contexto de la historia del continente. Por tanto, es difícil estar de acuerdo con el profesor Stengers de que pueda existir "una entidad 
política surgida en suelo africano por la exclusiva voluntad de los europeos” (Stengers, 1969, p. $26 \mathrm{I})^{5}$. Porque incluso en el Congo, el periodo colonial sólo representa un episodio en una larga historia llena de acontecimientos.

\section{Referencias}

Ade Ajayi, J.F. (1965). Christian missions in Nigeria, I84 I-I89I: the making of a new élite. Londres: Longmans.

Ade Ajayi, J.F. (1968). The continuity of African institutions under colonialism. En Ranger, T. O. (Ed.). Emerging themes in African history: proceedings of the International Historical Conference held at Dar es Salam. October 1965 (PP. XX-XX). Nairobi: East Africa Publishing House.

Ade Ajayi, J.F. (1969). Colonialism: An Episode in African History. En Gann, L. H. y Duignan, P. (Eds.). Colonialism in Africa 1870-1960.Vol. I.The History of Politics of Colonialism 1870-1914. Cambridge: Cambridge University Press.

Ade Ajayi, J.F., y Smith R.S. ( 1 964). Yoruba warfare in the nineteenth century. Cambridge: Cambridge University Press.

Colson, E. (1969). African society at the time of the scramble. En Gann, L. H. y Duignan, P. (Eds.). Colonialism in Africa 1870-1960.Vol. I.The History of Politics of Colonialism 1870-1914. Cambridge: Cambridge University Press.

Groves, C. P. (1969). Missionary and humanitarian aspects of imperialism from 1870 a 19|4. En Gann, L. H.y Duignan, P. (Eds.). Colonialism in Africa 1870-1960.Vol. I.The History of Politics of Colonialism I870-1914. Cambridge: Cambridge University Press.

Flint, J. E. (1969). Nigeria: the colonial experience from 1880 to 19|4. En Gann, L. H.y Duignan, P. (Eds.). Colonialism in Africa 1870-1960.Vol. I.The History of Politics of Colonialism 1870-19|4. Cambridge: Cambridge University Press.

Hargreaves, J. D. (1969).West African states and the European conquest. En Gann, L. H.y Duignan, P. (Eds.). Colonialism in Africa 1870-1960. Vol. I. The History of Politics of Colonialism 1870-1914. Cambridge: Cambridge University Press.

Johnston, Sir . H. (I899). History of Colonization of Africa by alien races. Cambridge: Cambridge University Press (2 ${ }^{\mathrm{a}}$ edición, 1913; reimpresión, 1930).

Jones, G.I. (1963). The trading states of the Oil Rivers: a study of political development in Eastern Nigeria. Londres: Oxford University Press.

Lagos Observer (19.02.1885). Lagos Observer 4 (2).

Stahl, K. M. (1964). History of the Chagga people of Kilimanjaro. Londres: Mouton.

Stengers, J. (1969). The Congo Free State and the Belgian Congo before 19I4. En Gann, L. H. y Duignan, P. (Eds.). Colonialism in Africa 1870-1960. Vol. I. The History of Politics of Colonialism 1870-1914. Cambridge: Cambridge University Press.

\footnotetext{
5 "Uno buscaría en vano por una subestructura africana o cualquier base autóctona de este estado tal como aparece a finales del siglo XIX...
} Sus orígenes deben ser buscado completamente en la voluntad de un hombre". 
Relaciones Internacionales Número 44 • Junio 2020 - Septiembre 2020 Grupo de Estudios de Relaciones Internacionales

Universidad Autónoma de Madrid 


\section{RELACIONES INTERNACIONALES}

Revista académica cuatrimestral de publicación electrónica Grupo de Estudios de Relaciones Internacionales (GERI)

Universidad Autónoma de Madrid, España

https://revistas.uam.es/relacionesinternacionales

ISSN 1699 - 3950

f facebook.com/RelacionesInternacionales

3. twitter.com/RRInternacional

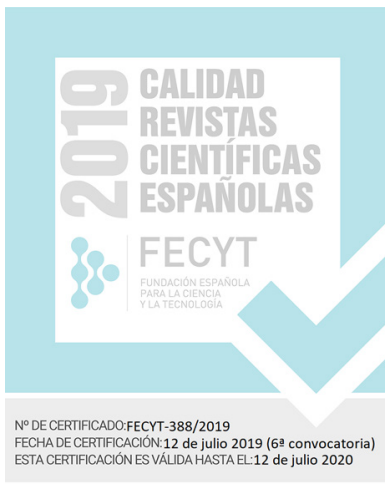

